\title{
Load dependence of cardiac output in biventricular pacing: Left ventricular volume overload in pigs
}

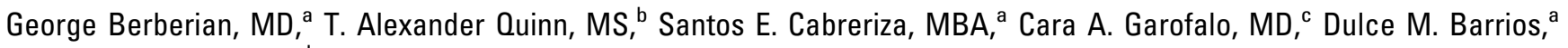
Alan D. Weinberg, MS, ${ }^{d}$ and Henry M. Spotnitz, MD ${ }^{a}$

Objective: Previous work from our laboratory has demonstrated that optimization of biventricular pacing is load dependent. Cardiac output was maximized with a ventricular-ventricular delay of +40 milliseconds (right ventricle-first pacing) during right ventricular pressure overload and with a ventricular-ventricular delay of -40 milliseconds (left ventricle-first pacing) during right ventricular volume overload. We hypothesized that a model of left ventricular volume overload would also have specific timing requirements during biventricular pacing for optimization of cardiac output.

Methods: After median sternotomy in 6 anesthetized pigs, complete heart block was induced by ethanol ablation. A conduit was grafted from the left ventricle to the left atrium to produce left ventricular volume overload. An ultrasonic flow probe was placed around the conduit to measure retrograde flow that averaged $50 \%$ of cardiac output. During epicardial atrial tracking DDD biventricular pacing, atrioventricular delay was varied between 60 and 270 milliseconds in 30-millisescond increments for 20-second intervals. After determination of optimum atrioventricular delay, ventricular-ventricular delay was varied in 20-millisecond increments from +80 to -80 milliseconds for 20 -second intervals.

Results: Ventricular-ventricular delays had no significant effect on cardiac output with the graft clamped (control). With the graft unclamped, however, there was a statistically significant $(P=.0001$ by repeated-measures analysis of variance) trend toward higher cardiac output with right ventricle-first pacing.

From the Departments of Surgery, ${ }^{\text {a }}$ Biomedical Engineering, ${ }^{\mathrm{b}}$ Pediatrics, ${ }^{\mathrm{c}}$ and Biostatistics, ${ }^{\mathrm{d}}$ Columbia University, College of Physicians and Surgeons, New York, NY.

Supported in part by the National Heart, Lung and Blood Institute of the National Institutes of Health (grant RO1 HL 48109 to Dr Spotnitz) and in part by the Department of Surgery, Columbia University College of Physicians and Surgeons.

Received for publication April 11, 2005; revisions received Sept 5, 2005; accepted for publication Sept 13, 2005.

Address for reprints: Henry M. Spotnitz, MD, Department of Surgery, Columbia College of Physicians and Surgeons, $622 \mathrm{~W}$ 168th St, PH 14-103, New York, NY 10032 (E-mail: hms2@columbia.edu).

J Thorac Cardiovasc Surg 2006;131:666-70 $0022-5223 / \$ 32.00$

Copyright $\odot 2006$ by The American Association for Thoracic Surgery

doi:10.1016/j.jtcvs.2005.09.017
Conclusions: Right ventricle-first pacing in swine significantly increased cardiac output during acute left ventricular volume overload, but not during the control state. Understanding load-specific pacing requirements will facilitate the development of perioperative temporary biventricular pacing for acute heart failure.

$\mathrm{O}$ ur laboratory has been studying biventricular pacing (BiVP) as therapy for acute heart failure in experimental animals. We have identified specific timing requirements during BiVP for optimization of hemodynamics in 2 ventricular pressure- and volume-overload models. These studies have shown that cardiac output (CO) was maximized with a ventricular-ventricular delay (VVD) of +40 milliseconds (right ventricle [RV]-first pacing) in a swine model of critical pulmonic stenosis with RV pressure (RVP) overload. ${ }^{1}$ In another swine model of acute tricuspid insufficiency with RV volume overload, $\mathrm{CO}$ was maximized with a VVD of -40 milliseconds (left ventricle [LV]-first pacing). ${ }^{2}$

Clinical trials have confirmed that BiVP can narrow the QRS complex and improve exercise capacity and quality of life, possibly leading to reduced hospitalization in patients with dilated cardiomyopathy and intraventricular conduction delays. ${ }^{3,4}$ Recent studies have also shown that the use of VVD in sequential BiVP improves stroke volume compared with simultaneous BiVP in patients with symptom- 


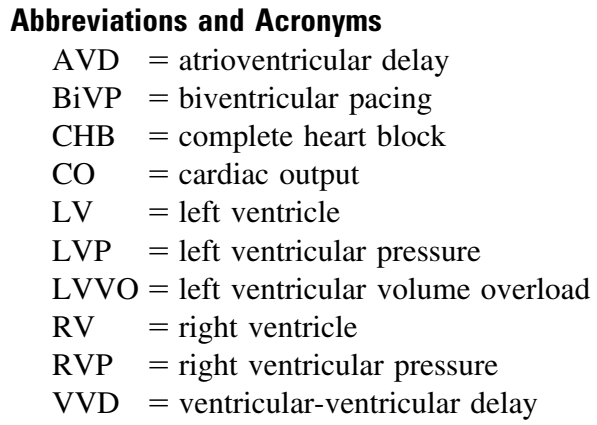

atic heart failure and prolonged QRS duration. ${ }^{5}$ However, the optimal VVD is highly variable from patient to patient and may change dynamically within each patient. This difference may reflect variable effects of right-sided and leftsided pressure or volume overload. In this study, we hypothesized that a model of LV volume overload (LVVO) would also have specific timing requirements during BiVP for optimization of $\mathrm{CO}$.

\section{Materials and Methods}

All animals received humane care in compliance with the Principles of Laboratory Animal Care developed by the Institute of Laboratory Animal Resources and the Guide for the Care and Use of Laboratory Animals prepared by the Institute of Laboratory Animal Resources and published by the National Institutes of Health (National Institutes of Health Publication no. 85-23, revised 1985).

\section{Surgical Preparation}

Six domestic pigs (40-45 kg) were anesthetized with ketamine hydrochloride (20 mg/kg intramuscularly), xylazine hydrochloride $(0.5 \mathrm{mg} / \mathrm{kg}$ intramuscularly), and atropine sulfate $(2 \mathrm{mg} / \mathrm{kg}$ intramuscularly). Pigs were intubated and mechanically ventilated;

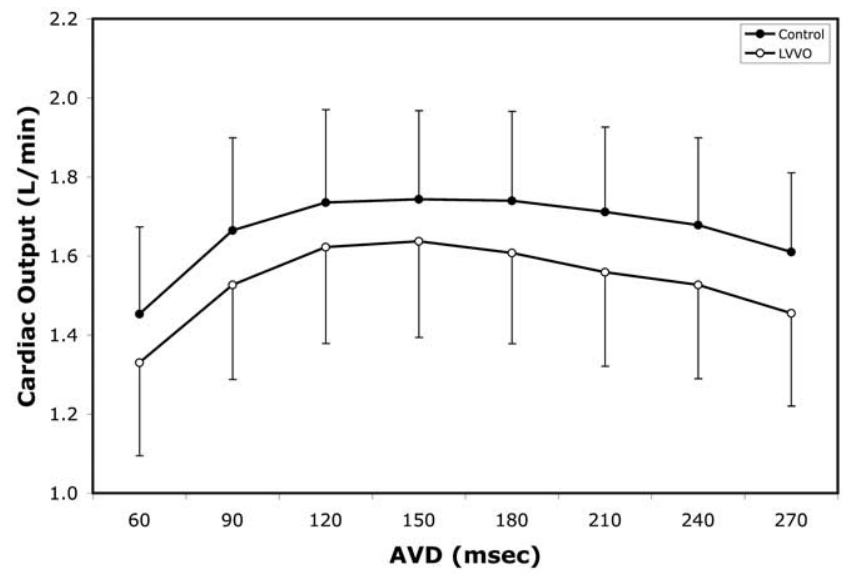

Figure 1. The effect of atrioventricular delay (AVD) on cardiac output in both control and left ventricular volume overload (LVVO) states.

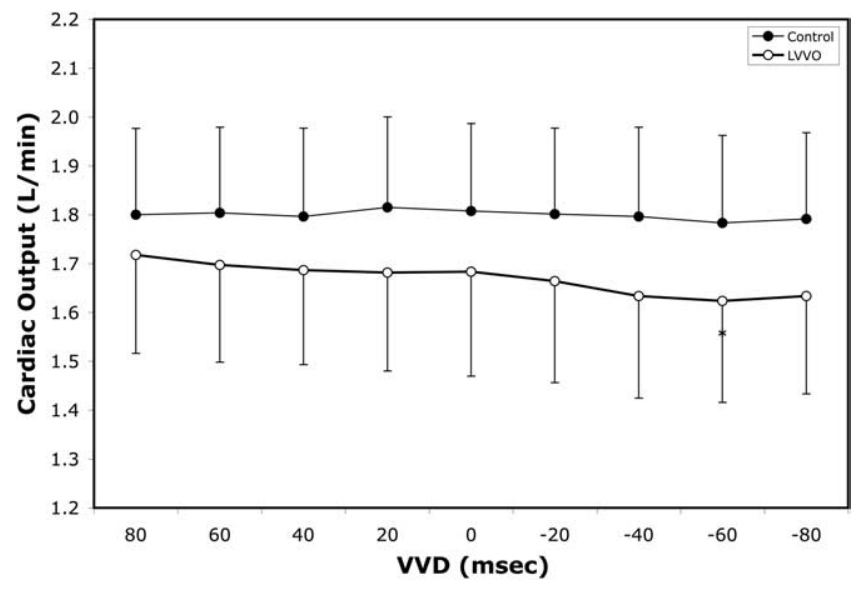

Figure 2. The effect of ventricular-ventricular delay on cardiac output in both control and left ventricular volume overload (LVVO) states.

arterial blood gas values were maintained within physiologic norms. Anesthesia was maintained with inhaled isoflurane $(1.5 \%$ $2 \%$ ) in oxygen. An 18-gauge angiocatheter was placed in an ear vein for an intravenous infusion of $0.9 \%$ saline. Electrocardiogram leads were attached to the limbs, and the left femoral artery was instrumented with a 20-gauge catheter attached to a pressure transducer to measure arterial pressure. After median sternotomy and longitudinal pericardiotomy, a lidocaine bolus $(3 \mathrm{mg} / \mathrm{kg}$ intravenously) was given, and a lidocaine drip was started at 50 $\mu \mathrm{g} \cdot \mathrm{kg}^{-1} \cdot \mathrm{min}^{-1}$ to suppress arrhythmias. A $24-\mathrm{mm}$ real-time ultrasonic flow probe (Transonic Systems Inc, Ithaca, NY) was placed on the ascending aorta. Temporary bipolar epicardial pacing leads (Medtronic Inc, Minneapolis, Minn) were placed on the right atrium, the anterior surface of the $\mathrm{RV}$, and the obtuse margin of the LV. Pacing wires were connected to a temporary external pacemaker (InSync III; Medtronic).

Once proper function of the pacemaker leads was confirmed, complete heart block (CHB) was established by injection of $0.5-\mathrm{mL}$ aliquots of $100 \%$ ethanol into the region of the bundle of His at the base of the aorta. The cumulative amount of ethanol required to establish $\mathrm{CHB}$ ranged from 0.5 to $1 \mathrm{~mL}$. After $\mathrm{CHB}$ was established, a segment of thoracic aorta harvested from another animal and stored in lactated Ringer and 2\% formalin solution was rinsed and then used as a graft. Modified right-angle aortic arch cannulas (6.5-mm Sarns; 3M Health Care, Ann Arbor, Mich) were secured to each end of the graft. Animals were heparinized with $300 \mathrm{IU} / \mathrm{kg}$ intravenously, and the metal cannulas were introduced sequentially through 4-0 Prolene purse-string sutures (Ethicon Inc, Somerville, NJ): first into the LV apex and then, after confirmation of vigorous flow, into the left atrium. Calibrated 5F micromanometer catheters (Millar Instruments, Houston, Tex) were placed in both the RV and LV through purse-string sutures. An ultrasonic flow probe was placed around the conduit to measure flow that averaged $50 \%$ of CO.

During atrial tracking DDD, BiVP was initiated at a heart rate of 90 beats per minute in all animals. Atrioventricular delay 
TABLE 1. Table showing the optimal and minimal ventricular-ventricular delays for each individual experiment

\begin{tabular}{lcccccc}
\hline Experiment no. & OPT/MIN VVD & RVP $\max$ (OPT) & RVP $\max$ (MIN) & LVP $\max$ (OPT) & LVP $\max$ (MIN) & \% Graft flow \\
\hline 1 & $+80 /-40$ & 18.4 & 17.3 & 59.5 & 57.4 & 61 \\
2 & $+80 /-60$ & 69.4 & 20.3 & 52.5 & 52.4 & 54 \\
3 & $+80 /-60$ & 20.0 & 19.8 & 51.0 & 50.2 & 46 \\
4 & $+80 /-60$ & 17.8 & 17.3 & 56.1 & 58 & 68 \\
5 & $0 /-60$ & 20.3 & 20.0 & 76.4 & 69.2 & 32 \\
6 & $+80 /-80$ & 23.1 & 23.6 & 66.6 & 67.9 & 40 \\
Mean & - & 28.2 & 19.7 & 60.3 & 59.2 & 50 \\
\hline
\end{tabular}

$L V P$, Left ventricular pressure; $M I N$, minimal ventricular-ventricular delay; $O P T$, optimal ventricular-ventricular delay; $R V P$, right ventricular pressure; $V V D$, ventricular-ventricular delay; max, maximal.

(AVD) was increased from 60 to 270 milliseconds and then decreased to 60 milliseconds in 30-millisecond increments for 20 -second intervals. After determination of the optimal AVD, VVD was decreased from +80 milliseconds (RV-first pacing) to -80 milliseconds (LV-first pacing) and was then increased to +80 milliseconds in 20-millisecond increments for 20-second intervals. At each pacing setting, electrocardiogram, arterial pressure, and aortic flow velocity were measured in the control state with the graft clamped and during LVVO with the graft open. Animals were humanely put to death at the conclusion of the experiment.

\section{Data Analysis}

Analog data for electrocardiogram, arterial pressure, RVP, LV pressure (LVP), aortic flow velocity, and graft flow velocity were sampled and transferred through a 16-channel analog-to-digital converter (MacLab; ADInstruments Inc, Milford, Mass) to a personal computer (iMac; Apple Computer, Cupertino, Calif). CO was determined for each experimental phase by integrating aortic flow velocity over time during a single complete respiratory cycle free of arrhythmia within each 20-second interval. Peak RVP and LVP for the optimal and minimal VVDs were determined for each of 8 beats within the corresponding respiratory cycle by integrating the corresponding pressure over time and were then averaged together.

For quantification of mechanical interventricular synchrony, 2 previously described measures were calculated from the RV and LVP signals at the corresponding VVDs that yielded the best and worst $\mathrm{CO}$ measurements. ${ }^{6}$ The first measure estimates the delay in milliseconds between the RV and LV contraction phases by shifting the upslope of the 2 normalized pressure curves in time until the cross-correlation coefficient between the signals reaches its maximal value (RVP-LVP upslope time shift, $T_{u p}$ ). The second measure is the area of the normalized RVP-LVP diagram, with a loop area of 0 indicating complete synchrony and a maximum area of 1 indicating complete asynchrony (RVP-LVP loop area, $A_{P P}$ ). $A_{P P}$ expresses asynchrony based on the pressure during the complete cardiac cycle, whereas $T_{u p}$ is restricted to the contraction phase. Both indices are positive for earlier RV than LVP and are equal to 0 for mechanical synchrony.

Intraventricular asynchrony was evaluated echocardiographically by using digitally acquired short-axis images of the LV at the level of the papillary muscles in 5 of 6 animals. Anatomical M-mode analysis on a GE Vivid 7 Cardiovascular Ultrasound System (GE Medical Systems, Milwaukee, Wis) allowed measure- ment of the shortest interval between the maximal systolic displacement of the interventricular septum and the LV posterior wall (septal-posterior wall motion delay). ${ }^{7}$ Positive values indicate maximal displacement of the septum preceding that of the free wall. Negative values indicate maximal displacement of the free wall preceding that of the septum. For each VVD setting, wall motion delay measurements were performed for 3 end-expiratory beats, and the values were averaged. We then compared wall motion delay results for the corresponding VVDs that yielded the best and worst $\mathrm{CO}$ measurements.

A statistical modeling procedure (described in the following section) was used to determine the effects of AVD and VVD on CO. To determine the effect of AVD on CO, VVD was kept at 0 , and the AVD was changed while aortic flow velocity was recorded.

\section{Statistical Methods}

For analyzing $\mathrm{CO}$, a 2-factor analysis of variance with repeated measures on both factors (AVD/VVD and LVVO vs control) was used. Because of the repeated-measures nature of the data for graft flow, a mixed-model method (PROC MIXED; SAS; SAS Institute Inc, Cary, NC) for a blocked design was used for both AVD and VVD experiments. A paired $t$ test was used to compare both peak RVP and LVP at the optimal and minimal VVDs, as well as indices for both interventricular and intraventricular synchrony. All data were analyzed by using SAS software.

\section{Results}

There was a statistically significant effect of AVD on CO in both the control and LVVO states, as presented in Figure 1 $(P=.0001)$. The optimal AVD in both control and LVVO was 150 milliseconds. The average effect of VVD on CO is presented in Figure 2. In the control state with the graft clamped, there was no statistically significant effect. During LVVO with the graft unclamped, CO improved with positive VVD $(P=.0001)$. Table 1 shows that the optimal VVD during LVVO was +80 milliseconds (RV-first pacing) in 5 animals and was 0 milliseconds in 1 animal. Both mean peak RVP and mean peak LVP were higher at optimal VVD than at minimal VVD. These differences were not significant. Figure 3 shows the effect of AVD on graft flow during LVVO $(P<.0001)$. There was no statistically significant 


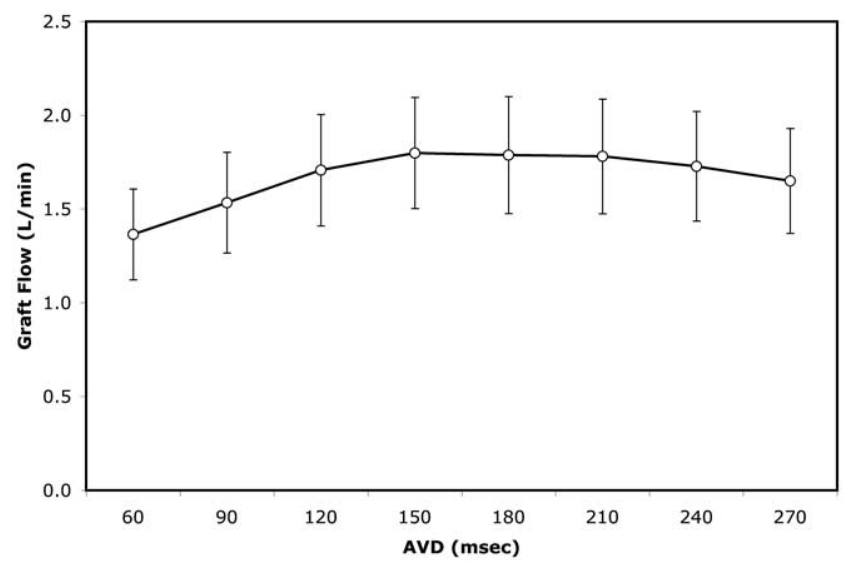

Figure 3. The effect of atrioventricular delay (AVD) on left ventricular-to-left atrial graft flow.

effect of VVD on graft flow, as presented in Figure 4. BiVP at optimum VVD significantly improved both indices of interventricular synchrony. While still asynchronous, optimum compared with minimum VVD settings gave values of $10.35 \pm 2.80 \mathrm{~ms}$ versus $-29.38 \pm 2.66 \mathrm{~ms}$ for $T_{u p}(P=$ $.00002)$ and $0.14 \pm 0.05$ versus $-0.25 \pm 0.03$ for $A_{P P}(P=$ .00009 ; Figure 5). Septal-posterior wall motion delay analysis showed a highly significant difference between optimal and minimal VVD settings: 45.4 milliseconds versus -72.4 milliseconds $(P=.0002$; Figure 6$)$.

\section{Discussion}

These results indicate that BiVP with optimized VVD in swine enhances depressed LV function in the setting of acute LVVO. The data provide further evidence that optimized timing of BiVP in the setting of acute heart failure is load dependent. Improved ventricular function is reflected

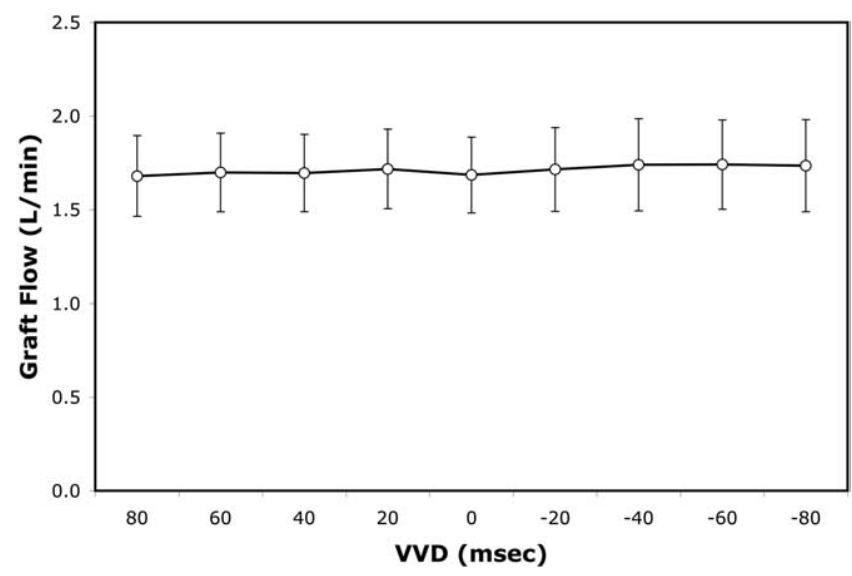

Figure 4. The effect of ventricular-ventricular delay on left ventricular-to-left atrial graft flow.

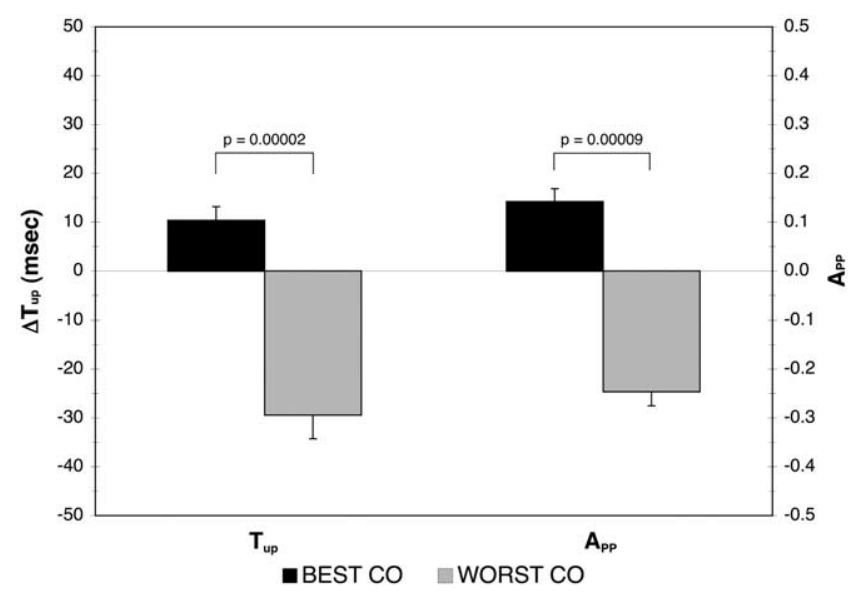

Figure 5. Indices of mechanical interventricular synchrony at ventricular-ventricular delays yielding the best and worst cardiac output (CO) measurements. $A_{P P}$ expresses asynchrony based on the pressure during the complete cardiac cycle, whereas $T_{u p}$ is restricted to the contraction phase. Both indices are positive for earlier right ventricular than left ventricular pressure and are equal to $\mathbf{0}$ for mechanical synchrony.

in $\mathrm{CO}$ and stroke volume. In this study, we focused primarily on the effect of AVD and VVD on CO. Physiologically, lead placement may be as or more important to optimizing ventricular function. Our laboratory is currently investigating the importance of lead location during BiVP for optimization of $\mathrm{CO}$.

The relationship between AVD and $\mathrm{CO}$ is related to chamber mechanics through optimization of ventricular filling. ${ }^{8}$ An excessively long or short interval is known to result

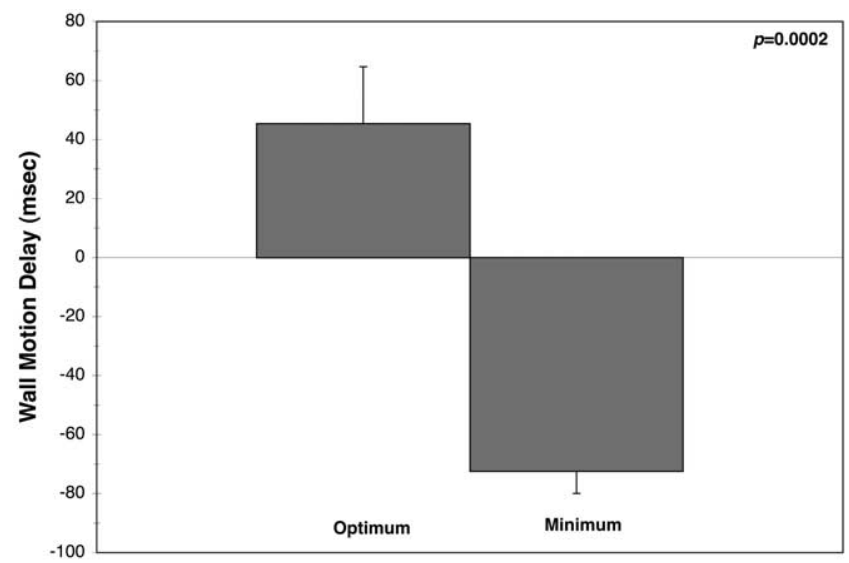

Figure 6. Septal-posterior wall motion delay analysis showing a highly significant difference between optimal and minimal ventricular-ventricular delay settings. Optimal ventricular-ventricular delay settings were uniformly associated with positive wall motion delays. 
in suboptimal chamber filling that contributes to mitral regurgitation. ${ }^{9}$ Effects of AVD on $\mathrm{CO}$ in this study support this premise by demonstrating that extreme values (60 and 270 milliseconds) were detrimental to $\mathrm{CO}$ in both the control and the LVVO states.

This study also examined the effect of VVD on CO during acute LVVO. Our previous study of BiVP during acute RVP overload demonstrated that the optimal VVD was negative (LV-first pacing). ${ }^{2}$ In this experiment, $\mathrm{RV}$-first pacing at a VVD of +80 milliseconds provided the maximum $\mathrm{CO}$ in 5 of our 6 animals. This variability in 1 animal may reflect problems with our model, such as possible ethanol-related myocardial injury or injury to the LV during placement of the conduit.

Our laboratory is in the process of defining mechanisms for the effects of VVD on CO. ${ }^{10}$ In a recent study of LV and RVP development in our experimental model of pulmonic stenosis, optimized pacing was associated with statistically significant benefits in interventricular synchrony. ${ }^{11}$ In this study, although both mean peak RVP and mean peak LVP were higher at the optimal VVD, these differences were not significant. BiVP at the optimal VVD did, however, significantly improve indices of interventricular synchrony. Septal-posterior wall motion delay analysis also showed a significant difference between optimal and minimal VVD settings. Optimal VVD settings were uniformly associated with positive wall motion delays, and this suggests that displacement of the septum preceding that of the free wall is more beneficial in this model of LVVO. Future studies investigating the mechanism of BiVP optimization are clearly needed and should define geometric changes by means of conductance.

Our results continue to support further development of BiVP for clinical application, especially with regard to the notion of load dependence. Although acute studies in laboratory animals with otherwise normal myocardium and a specific pathologic loading state may not be directly transferable to a clinical setting, there are data to suggest that pathologic loading conditions may play a critical role during BiVP in isolated patients. ${ }^{12}$ The most favorable results are likely to be obtained with a BiVP-optimization protocol for each patient. Clinical studies are indicated to identify hemodynamic trends for patients in response to specific pathologic loading conditions.

\section{References}

1. Rabkin DG, Cabreriza SE, Curtis LJ, Mazer SP, Kanter JP, Weinberg $\mathrm{AD}$, et al. Load dependence of cardiac output in biventricular pacing: Right ventricular pressure overload in pigs. J Thorac Cardiovasc Surg. 2004;127:1713-22.

2. Rabkin DG, Curtis LJ, Cabreriza SE, Weinberg AD, Spotnitz HM. Load dependence of cardiac output in biventricular pacing: right ventricular volume overload in pigs. J Thorac Cardiovasc Surg. 2004; 128:98-102.

3. Cazeau S, Leclercq C, Lavergne T, Garrigue S, Bailleul C, Daubert JC, et al. Multisite Stimulation in Cardiomyopathies (MUSTIC) Study Investigators. Effects of multisite biventricular pacing in patients with heart failure and intraventricular conduction delay. $N$ Engl J Med. 2001;344:873-80.

4. Abraham WT. Rationale and design of a randomized clinical trial to access the safety and efficacy of cardiac resynchronization therapy in patients with advanced heart failure: the Multicenter inSync Randomized Clinical Evaluation (MIRACLE). J Card Fail. 2000;6:369-80.

5. Mortensen PT, Sogaard P, Mansour H, Ponsonaille J, Gras D, Lazarus A, et al. Sequential biventricular pacing: evaluation of safety and efficacy. Pacing Clin Electrophysiol. 2004;27:339-45.

6. Verbeek XA, Vernooy K, Peschar M, van der Nagel T, van Hunnik A, Prinzen FW. Quantification of interventricular asynchrony during LBBB and ventricular pacing. Am J Physiol Heart Circ Physiol. 2002;283: H1370-8.

7. Pitzalis MV, Iacoviello M, Romito R, Massari F, Rizzon B, Luzzi G, et al. Cardiac resynchronization therapy tailored by echocardiographic evaluation of ventricular asynchrony. J Am Coll Cardiol. 2002;40: 1615-22.

8. Wish M, Fletcher RD, Gottdiener JS, Cohen AI. Importance of left atrial timing in the programming of dual-chamber pacemakers. Am J Cardiol. 1987;60:566-71.

9. Brecker SJ, Xiao HB, Sparrow J, Gibson DG. Effects of dual-chamber pacing with short atrioventricular delay in dilated cardiomyopathy. Lancet. 1992;340:1308-12.

10. Rabkin DG, Cabreriza SE, Curtis LJ, Quinn TA, Weinberg AD, Hordof $\mathrm{AD}$, et al. Mechanisms of optimized biventricular pacing in pulmonary stenosis: effects on left ventricular geometry in swine. Pacing Clin Electrophysiol. 2004;27:1060-71.

11. Quinn TA, Berberian G, Cabreriza SE, Kanter JP, Curtis LJ, Spotnitz HM, et al. Ventricular systolic mechanics and synchrony improve with biventricular pacing in critical pulmonary stenosis. Circulation. 2004; 110(suppl):III-32.

12. Berberian G, Kanter JP, Quinn TA, Spotnitz HM. Optimized perioperative biventricular pacing in setting of right heart failure. Europace. 2005;7:385-7. 